\title{
Status Quo of Evidence-Based Design Research and Its Research Trends in Korean Healthcare Architecture
}

\author{
한국 의료건축연구의 근거기반설계에 대한 최근 연구동향 \\ Kim, Duk-Su* 김덕수 | Kwun, Joon-Bum** 권준범
}

\begin{abstract}
Purpose: The purpose of this study is to provide understandings about EBD research trends in Korean healthcare architecture research. Method: The systematic literature review was conducted, while comparing the characteristics of research papers in Journal of Korea Institute of Healthcare Architecture with EBD related research papers among them. Results: It is found that the difference of research method utilized between healthcare research in general and EBD research. Specifically, a research method most utilized in general is a field study. In EBD research, however, literature review is most frequently used. Implications: This study can be used as a reference point for further development of EBD research in Korean healthcare architecture. In addition, research results can be used as a communication tool with healthcare specialists such as doctors and consultants in a healthcare project team. Thus, this study is anticipated to play a preliminary role for the development of healthcare architecture design in Korea.
\end{abstract}

Keywords Evidence-based design, Research trends, Healthcare Architecture 주 제 어 근거기반설계, 연구동향, 의료건축

\section{Introduction}

\subsection{Background and Objective}

프로페션으로서의 건축이 직면하고 있는 문제점에 대한 지 적은 건축지식의 이중적 속성에 기인하는 건축의 직능적 정 체성에 대한 혼란과 자본주의 정치경제 시스템에서 프로페션 으로서의 건축이 수행하는 사회적 역할의 축소에 대한 논의 로 수렴된다. 가장 오래된 프로페션 중의 한 유형인 건축이 갖 는 사회적 위상의 축소에 대한 논의는 건축 직능의 발전 방안 에 관한 고민으로 수렴되며 건축전문 출판물에서 지속적으로 발견되는 테마이다.

특히, 건축의 모순적인 지식 속성이 야기하는 갈등관계에 대한 지속적 논의가 있다. 하나는 건축의 변화를 예술에서 기 인하는 자율성의 발현으로 파악하는 것이고, 또 다른 하나는

* Associate Professor, Ph.D, Department of Architecture, Hanbat National University (Primary author: dsk@hanbat.ac.kr)

** Associate Professor, Ph.D, Department of Architecture, Sejong University (Corresponding author: joonkwun@sejong.ac.kr)
대응이론의 관점에서 원인과 결과의 대상적 관계를 인과관계 로 파악하는 과학으로서의 건축관이다. 건축의 과학적 접근은 주로 건축환경이나 구조설계와 같은 기술 분야에서 집중적으 로 이루어지고 있다. 건축 유형 중에서는 의료건축 설계분야 에서 연구의 타당도와 신뢰도를 보장하기 위한 절차적 엄밀 성이 강조되는 과학적 연구방법이 가장 많이 적용되고 있다. 즉 건축물의 해석과 관련되는 복수적 의미의 파악보다는 설 계된 건축물이 기대되는 기능을 충실히 수행할 수 있는 가에 초점을 맞추는 건축물의 수행과 관련된 높은 수준의 일반성 획득을 목표로 한다. 그러나 한국 건축설계사무소에서는 상대 적으로 의료건축 설계의 전문화가 미비하고 건축대학에서도 학부과정에서 특화된 건축교육이 이루어지는 경우가 많지 않 다. 의료건축 설계 전문화와 전문교육의 미비에 기인하여 의 료건축 설계를 담당하고 있는 실무자들은 의료건축 설계의 지향점 설정과 문제해결에 필요한 전문지식의 속성 및 방법 론에 대한 혼란을 경험하게 된다. 세계적으로 높은 수준의 건 축설계 경쟁력을 갖고 있다고 평가받는 미국에서는 건축유형 에 따른 전문화가 번성해있다. 따라서 동일한 건축유형에 대 
한 반복적 설계가 가능하여 건축가 개인의 차원에서는 풍부 한 실무경험을 쌓아 전문성을 제고할 수 있고 건축설계사무 소의 차원에서는 전문지식을 사내에 집적할 수 있는 장점이 있다. 미국 건축설계교육에 국한하여 보더라도 집중적으로 필 요지식을 습득하도록 하기 위해 의료건축에 전문화된 교과과 정을 제공하고 전문교육프로그램 수료인증서를 수여하고 있 는 건축교육의 전문화 역시 발견된다(Moon, 2011: 7-14). 의 료건축 설계와 관련된 실무적 맥락의 변화에 대응하여, 의료 건축설계 전문 프로그램을 운영하고 있는 건축학과 교육의 강조점은 의료건축물의 목적과 클라이언트 및 사용자의 기대 를 만족시키기 위한 근거기반설계(Eevidence-Based Design: $\mathrm{EBD})$ 에 놓여있다. EBD 배경 중의 하나는 미국 의료서비스의 품질 저하에 대한 대응의 필요성이다. 미국의 통계자료를 보 면 한 해에 약 98,000 명이 의료사고로 사망하고 있다 (Lavizzo-Mourey, 2004). 이에 대해 건축분야에서는 효율적 인 의료건축 설계와 관리를 통해 위해환경과 위험요소의 감 소 및 통제, 사고와 부상 방지, 안전 유지, 환자 중심의 환경 구축, 그리고 환경 스트레스를 감소시켜 의료사고를 줄이기 위한 방안으로 $\mathrm{EBD}$ 를 활용하고 있다(The Joint Commission, 2009).

2003년 이후 미국의 의료건축 설계분야에서 공식적으로 통용되기 시작한 EBD의 개념은(Hamilton, 2003: 20), 2008년 이래 한국의료복지건축학회 논문집을 중심으로 국내 의료건 축설계 분야에 소개되고 있으나 아직 건축설계 실무나 학술 적 연구에 활발히 적용되지는 못하고 있다. 그 이유로는 $\mathrm{EBD}$ 의 발생기원, 개념, 증거의 유형과 수준, 방법 등에 대한 인식 이 명확히 정립되지 않은 것과 인과관계를 적시하는 공식화 된 증거만을 설계결정의 근거로 사용하는 설계과정이 건축가 의 창의성과 자율성을 침해한다는 인식에 기인한다고 본다. 이러한 문제의식에 의해 기획된 본 연구는 국내의 건축전문 학술지를 중심으로 하여 $\mathrm{EBD}$ 에 관련된 연구의 속성을 $\mathrm{EBD}$ 평가 틀에 의해 분석한다. 또한 건축이 프로페션으로 존립하 기 위해 수행해야 하는 사회적 역할은 대중의 건강, 안전, 복 지(Health, Safety, Welfare: HSW)를 보호하는 것이라고 할 때, $\mathrm{EBD}$ 는 이러한 프로페션으로서의 지향점을 예시하는 관점의 실무적 구현 노력으로 이해할 수 있다. 따라서 한국 의료건축 설계에 $\mathrm{EBD}$ 연구를 적용하기 위해 선행되어져야 하는 기초자 료 제공을 목적으로 하는 본 연구는 건축설계결정의 신뢰도 를 높이기 위한 방안으로 미국을 중심으로 활발히 전개되고 있는 $\mathrm{EBD}$ 연구 동향을 분석하여 건축계 내부의 연구자와 실 무건축가에게 $\mathrm{EBD}$ 의 개념에 대한 이해를 제공한다. 이를 통 해 근거기반실무(Evidence-Based Practice)의 중요성을 인정 하고 있는 의료 및 보건 분야의 전문가를 포함하기 때문에 다 학제적 속성을 갖게 되는 의료건축 프로젝트를 진행할 때 공 통적 언어를 사용하게 되어 의사소통 능력 제고와 결과적으
로 한국 의료건축 설계의 발전에 기여할 수 있는 기초자료의 역할을 기대한다.

\subsection{Methods of Research}

현재 미국의 의료건축설계를 중심으로 상당한 파급력을 갖 고 진행되고 있는 $\mathrm{EBD}$ 의 태동과 발전에 주목하는 본 연구는 체계적 문헌조사를 통해 미국을 중심으로 하여 2000년대 이 후로 활발히 논의되고 있는 $\mathrm{EBD}$ 의 발생 동기와 특성을 검토 한다. EBD 개념 정립을 위해 참조한 자료는 의료설계센터 (The Center for Health Design: CHD)의 출판물과 CHD가 시 행하는 근거기반설계 인증 및 자격(Evidence-Based Design Accreditation and Certification: EDAC) 프로그램 자료, 미국 의료건축가협회(American College of Healthcare Architects: $\mathrm{ACHA}$ ) 홈페이지 자료 및 부속 전문학술지의 논문, 그리고 기 타 근거기반의학(Evidence-Based Medicine: EBM)이나 EBD와 관련된 다양한 전문학술지(e.g., Health Environment Research \& Design Journal: HERD)의 게재논문을 포함한다. 상기의 연 구자료에서 추출한 $\mathrm{EBD}$ 증거의 위계적 분류체계를 분석의 틀 로 활용하는 본 연구는 국내 건축전문학술지에 게재된 $\mathrm{EBD}$ 관 련 연구논문을 구체적 분석의 단위로 설정한다. 따라서 국내 에서 이루어지고 있는 $\mathrm{EBD}$ 연구 현황을 의료복지건축학회논 문집과 대한건축학회논문집을 중심으로 분석하는 체계적 문 헌조사(systematic literature review)로 진행한다.

\section{Definition of Evidenced-Based Design}

\subsection{Evidence-Based Movement and the Emergence of Evidence-Based Design}

의료건축 설계분야를 중심으로 하여 건축에서 활발히 논의 되고 있는 $\mathrm{EBD}$ 는 의료건축 시장의 변화에 대한 적극적 대응 의 결과이다. 의료건축에 특화된 건축회사들은 그들의 클라이 언트인 의사나 병원 운영자들이 직면하고 있는 의료 실무의 맥락변화에 주목하고 있었다. EBD는 1972년 영국 의학계가 의사들의 교육적 배경이나 경험에 의지하여 환자를 치료하는 관습적 의료 실무관행이 환자들의 치유에 부정적 영향을 미 친다고 주장하며 제시한 근거기반모델(Evidence-Based Model) 에 그 시원을 둔다. 과거의 의료실무 관행에 대한 대안으로 제 시된 근거기반모델은 최신의 과학적 연구결과에 기반을 둔 의료행위를 강조하는 근거기반의학(Evidence-Based Medicine: $\mathrm{EBM})$ 으로 구체화되었다. 근거기반모델은 근거기반간호(EBP in Nursing: Evidence-Based Nursing)와 근거기반경영(EBMgt: Evidence-Based Management) 등 많은 전문분야에 도입되고 있다. 다양한 프로페션이 근거기반모델을 적용하여 전문적 권 위의 증진을 추구하는 추세에 영향 받아 건축에서도 $\mathrm{EBD}$ 가 대두되었다. 즉, 건축설계분야에서 $\mathrm{EBD}$ 의 개념화는 $\mathrm{EBM}$ 이라 
는 용어가 1992년부터 사용되기 시작한 이래(EBM Working Group, 1990: 2240-2245), 1996년에 내려진 EBM에 대한 다 음과 같은 정의에 직접적인 영향을 받아 이루어졌다(Stichler, 2008: 3). "근거기반의학은 환자의 치료를 위한 결정을 내리 기 위해 존재하는 최선의 증거를 양심적으로, 분명하게, 그리 고 신중하게 사용한다. EBM은 체계적 조사를 통해 개별적 의 료 전문지식과 최고의 가용 증거의 통합을 의미한다." $\mathrm{EBM}$ 과 유사한 맥락 하에 $\mathrm{EBD}$ 는 각각 개별적이고 고유한 특성을 갖는 프로젝트의 설계에 관해 전문적 클라이언트와 협력하여 중요한 의사결정을 내릴 시에 연구조사와 실무로부터 최선의 증거를 양심적으로, 분명하고, 신중하게 사용하는 과정으로 정의 내려진다. 즉, 의학이나 의료건축설계에서 전문적 결정 은 개인의 교육과 경험에 기반하여 이루어지는 것이 아니라 체계적 연구조사에 의해 획득한 최선의 증거에 기반을 두고 내려져야 한다(표 1).

[Table 1] Definitions of Evidence-Based Movement

\begin{tabular}{|c|c|}
\hline & Definition \\
\hline $\begin{array}{l}\frac{3}{0} \\
\stackrel{0}{0} \\
\frac{2}{3}\end{array}$ & $\begin{array}{l}\text { Evidence-based medicine is the conscientious, explicit, } \\
\text { and judicious use of current best evidence in making } \\
\text { decisions about the care of individual patients. The } \\
\text { practice of EB Medicine means integrating individual } \\
\text { clinical expertise with the best available evidence } \\
\text { from systematic research(Stichler, 2008: 3). }\end{array}$ \\
\hline 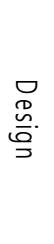 & $\begin{array}{l}\text { Evidence-based design is a process for the } \\
\text { conscientious, explicit, and judicious use of current } \\
\text { best evidence from research and practice in making } \\
\text { critical decisions, together with an informed client, } \\
\text { about the design of each individual and unique } \\
\text { project(Hamilton, 2006: 31). }\end{array}$ \\
\hline 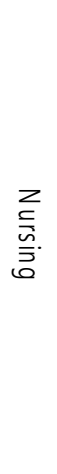 & $\begin{array}{l}\text { Evidence-based nursing is defined as the incorporation } \\
\text { of evidence from research and clinical practice plus } \\
\text { patient preferences into clinical decisions(Munhall, } \\
\text { 1998: 4-6). } \\
\text { Evidence-based practice is a professional approach to } \\
\text { practice that involves a deliberate, thoughtful use of } \\
\text { "best" available evidence in the management of } \\
\text { individual patients as well as groups of patients, and } \\
\text { "an approach that de-emphasizes ritual, isolated, and } \\
\text { unsystematic clinical experiences, ungrounded opinions, } \\
\text { and tradition as a basis for nursing practices"(Stetler, } \\
\text { 1998: 46). }\end{array}$ \\
\hline $\begin{array}{l}3 \\
3 \\
3 \\
00 \\
0 \\
0 \\
3 \\
0 \\
0 \\
0\end{array}$ & $\begin{array}{l}\text { Evidence-based management is about making decisions } \\
\text { through the conscientious, explicit, and judicious use } \\
\text { of four sources of information; practitioner expertise } \\
\text { and judgement, evidence from the local context, a } \\
\text { critical evaluation of the best available research } \\
\text { evidence, and the perspectives of those people who } \\
\text { might be affected by the decision(Briner et. al., 2009: } \\
\text { 19). }\end{array}$ \\
\hline
\end{tabular}

의학의 임상과 마찬가지로 건축실무도 전문지식의 획득과 운용과정에서 유사한 속성이 발견된다. 기초과학과 같이 새로 운 지식을 산출하는 전통적인 연구와 비교되는 $\mathrm{EBD}$ 의 특징은 새로운 지식의 창출보다는 설계 결정을 위해 기존의 지식을 체계적 절차에 의해 선별하여 사용하고 설계의 효율성을 검 증하는 점에 있다(Stichler, 2010, 10). 따라서 EBM과 마찬가 지로 $\mathrm{EBD}$ 실무에서도 어떤 수준의 증거가 유용한지에 대한 논의는 근거기반실무의 핵심이 되고 있다(Saitz, 2013: 1).

연구문헌에서 발견되는 $\mathrm{EBD}$ 의 효용은 다음과 같이 요약할 수 있다: (1) 전문적 클라이언트의 속성에 대한 대응과 설계 결정의 신뢰성 확보; (2) 마케팅 효용; 그리고 (3) 프로페셔널 리즘의 제고. 구체적으로 살펴보면, 첫째, 의료시설 설계에서 $\mathrm{EBD}$ 는 전문가로 구성되는 의료건축 클라이언트를 설득시킬 수 있는 설계 결정의 신뢰성을 증진시키는 효용이 있다. 다른 분야와 비교하여 병원 프로젝트는 클라이언트나 주요 사용자 가 되는 의사들이 건축가보다 병원에 대해 더 많이 알고 있다 고 인식하고 있기 때문에 건축가는 설계 결정의 자율성 침해 를 경험하고 있다. 병원 설계의 경우, 건축가의 설계 결정에 대한 신뢰도가 저하되는 가장 큰 이유로는 설계 결정의 타당 성을 객관적으로 설명할 수 있는 기준의 부족에 있다고 논의 된다(Kim; Shepley, 2008: 22). 근대 병원은 신뢰도 있는 치료 의 기능을 수행하기 위해 기계와 같은 정확성을 갖는 시스템 으로 진화해 왔으나, 기계와 같은 시스템은 치유환경에 대한 새로운 과학적 발견에 의한 병원 공간구조의 변화 요구, 의료 기술 발전에 기인하는 진단과 치료 방식의 변화, 인구 구성 및 질병 유형 등의 변화에 융통성 있게 대응하지 못하게 되어 더 이상 효율성, 예측성, 일관성 있는 결과를 산출하는 능력을 잃 어버리게 되었다. EBM의 방법과 절차에 직접적으로 영향을 받은 $\mathrm{EBD}$ 는 병원 사용자에게 미치는 소리, 조망, 색채, 공기, 빛 등의 영향에 대한 증거를 축적하며, 이러한 환경의 생리적 및 심리적 영향을 고려하는 새로운 병원을 설계할 수 있게 한 다. 즉 $\mathrm{EBD}$ 에 의해 치유환경이 필요로 하는 것을 조사하여 해 결하는 방안을 제시하여 의료건축 설계 결정에 대한 신뢰도 를 증진시키고 있다(Douglas et. al., 2011: 44).

둘째, 비즈니스 측면으로 보면 $\mathrm{EBM}$ 의 건축적 해석인 $\mathrm{EBD}$ 는 마케팅 효용을 갖고 있다. 의료서비스 분야에서 EBM의 적 극적 수용과 발전은 의료서비스 제공자들의 관심에 부합하려 는 현실적 동기가 크게 작용하고 있다. 미국국립보건원 (National Institute of Health: NIH)에서는 의료서비스의 품질 을 평가하고 관리하는 수단으로 $\mathrm{EBM}$ 을 적극 권장하고 있다. 연구자들은 $\mathrm{NIH}$ 의 연구비 수혜를 위해 EBM을 수용하고 있 고, 건축은 이러한 의료서비스 정책을 둘러 싼 맥락의 변화에 맞춰 병원 클라이언트의 시장경쟁력 강화 욕구를 충족시키기 위해 EBM을 건축설계에 적용하는 EBD 역량을 마케팅 수단으 로 활용하고 있다. 증가하는 병원간의 경쟁을 이겨내고 고객 
의 만족을 증진시키기 위해 병원들은 자신들이 최고의 의료 서비스를 제공한다는 메시지를 전달하여 강력한 브랜드를 창 조하려고 노력한다. 강력한 브랜드는 환자, 방문객, 의료 스태 프를 포함하는 이용자들의 만족도를 높이고 동시에 의료서비 스 수혜자들의 충성도를 높여 미래의 재방문을 가능하게 하 기 때문이다. 이러한 이유에 의해 병원들은 이용자들에게 긍 정적 경험을 제공하고 고품질의 의료서비스를 건축적으로 암 시하는 최고의 전시성 프로젝트들을 건설하고 있다(Noakes, 2009: 111).

마지막으로 $\mathrm{EBD}$ 는 건축의 프로페셔널리즘을 제고할 수 있 다. 역사적으로 건축가들은 경험과 감각, 병원 컨설턴트의 자 문, 규정과 클라이언트의 요구에 따라 의료시설을 설계해 왔 다. 그러나 $\mathrm{EBD}$ 의 도입에 의해, 건축가들은 디자인 결정의 타 당성을 설명할 수 있는 객관적 증거를 디자인 결정의 근거로 참고한다. 향후, 더욱 믿을 만한 연구 데이터와 신뢰도 높은 증거의 증가는 의료건축 설계 발전의 중요 동인이 될 것이며, 또한 설계결정에 이용자의 HSW를 보호할 수 있는 객관적 지 식을 적용하여 프로페션으로서의 건축이 사회에 미치는 직능 적 역할을 강화할 수 있게 된다(Lewis, 2008: 16). 이것은 라 이선스를 부여받은 프로페션으로서의 건축이 대중의 HSW를 보호하기 위한 진지한 노력의 일환을 예시하여 프로페션의 사회적 기능에 대한 대중의 신뢰에 응답하는 것이다 (Hamilton, 2003: 25).

\subsection{Types and Levels of Evidence}

$\mathrm{EBD}$ 증거의 수준은 내적 타탕도(Internal Validity)1)를 성취 할 수 있는 연구디자인의 속성에 따라 위계적으로 분류된다 (Pati, 2011: 61-70). 일반적으로 증거의 유형은 과학적 연구 의 질을 평가하는 주요 기준 중의 하나인 내적타당도의 성취 정도에 따라 $\mathrm{EBD}$ 에 적용할 수 있는 증거로서의 가치를 위계 적으로 평가받는다. 증거의 위계는 최고 수준인 1단계부터 6 단계까지 나눠진다(Stichler, 2010: 3-7)(표 2).

$\mathrm{EBD}$ 에 적용하는 가장 높은 위계를 갖는 증거는 복수의 무 작위 샘플링 통제연구(Random Control Treatments: RCTs)나 작위적 샘플링에 의한 연구물들의 체계적 분석조사 (Systematic Review), 다수의 실험연구(Experimental Research) 나 유사실험연구(Pseudo-Experimental Research)의 메타분석 (Meta-Analysis)2), 그리고 통합적 해석을 가능하게 하는 다수 의 질적 연구물을 종합한 연구물(Meta-Systhesis)이다. 기존의

1) 교육학용어사전의 정의에 따르면, 실험적 연구에 있어서 주어진 실험처치(experimental treatments)가 정말로 실험효과를 가져왔 느냐 하는 정도를 말한다. 이 내적 타당도에서는 주어진 실험처 치가 이 실험에서 정말로 어느 정도 실험효과를 가져왔느냐에 대 한 질문의 답이 된다.

2) 단일 주제를 대상으로 하는 다수의 연구들을 체계적으로 요약하 는 문헌연구의 한 방법
유사 연구들과 비교했을 때 신뢰도 높은 결과를 산출한 실험 연구나 유사실험연구는 두 번째로 높은 위계를 갖고 있다. 반 면에 $\mathrm{EBD}$ 에 적용할 때 가장 낮은 위계를 갖는 증거로는 경제 적 이해당사자가 되는 생산업체나 컨설턴트들의 추천을 언급 하고 있다. 즉, 과학적 연구디자인의 준수에 의해 연구의 타당 도와 신뢰도를 획득했다고 인정받는 연구 결과물들의 체계적 분석조사는 $\mathrm{EBD}$ 증거로서 최고의 위계를 인정받고 있다.

[Table 2] Levels of Evidence for Healthcare Design

\begin{tabular}{l|l}
\hline Level 1 & $\begin{array}{l}\text { Systematic reviews of multiple randomized } \\
\text { controlled trials (RCTs) or nonrandomized } \\
\text { studies; meta-analysis of multiple experimental } \\
\text { or quasi-experimental studies; meta-synthesis } \\
\text { of multiple qualitative studies leading to an } \\
\text { integrative interpretation }\end{array}$ \\
\hline level 2 & $\begin{array}{l}\text { Well-designed experimental (randomized) and } \\
\text { quasi-experimental (nonrandomized) studies } \\
\text { with consistent results compared to other, } \\
\text { similar studies }\end{array}$ \\
\hline Level 3 & $\begin{array}{l}\text { Descriptive correlational studies, qualitative } \\
\text { studies, integrative or systematic reviews of } \\
\text { correlational or qualitative studies, or RCT or } \\
\text { quasi-experimental studies with inconsistent } \\
\text { results compared to other, similar studies }\end{array}$ \\
\hline Level 4 & $\begin{array}{l}\text { Peer-reviewed professional standards or } \\
\text { guidelines with studies to support re- } \\
\text { commendations }\end{array}$ \\
\hline Level 5 & $\begin{array}{l}\text { Opinions of recognized experts, multiple case } \\
\text { studies }\end{array}$ \\
\hline Level 6 & $\begin{array}{l}\text { Recommendations from manufacturers or } \\
\text { consultants who may have a financial interest } \\
\text { or bias }\end{array}$ \\
\hline
\end{tabular}

또한 건축설계의 실무적 맥락에서도 의료건축 설계에 적용 할 수 있는 증거의 질과 건축설계 결정 근거로서의 신뢰도가 매트릭스로 제안된다(Hamilton, 2011: 125). 제시된 매트릭 스는 과학적 연구 결과물의 증거력을 가장 높게 평가하며, 다 음으로 상관성 연구(Correlation Study), 설계 사례연구들의 분석, 전문가의 의견이나 실무경험의 순으로 증거력의 수준을 평가하고 있다. 한편, $\mathrm{EBD}$ 를 실무에 도입하는 의료건축 전문 건축가의 수준은 4가지로 분류된다(Hamilton, 2003: 19-22) (표 3). 1단계 실무자는 자료를 읽고 해석하는 단계를 말하고, 2 단계에 있는 실무자는 1 단계 과정을 통해 획득한 자료를 활 용해 디자인에 의해 발생하는 예상된 결과에 대해 가설을 설 정하고 실제로 그 결과를 측정한다. 3단계 실무자는 디자인에 의해 발생한 결과를 공개적으로 발표한다. 마지막 4단계에서 는 전문가들에 의한 심사를 필요로 하는 전문학술지에 연구 결과를 게재한다. 이러한 근거기반 실무의 위계에 따른 수준 별 활동의 예시를 통해 건축가의 $\mathrm{EBD}$ 역량이 위계적으로 평 가된다. 
[Table 3] Four Levels of Evidence-Based Practice

\begin{tabular}{|c|c|c|c|c|c|}
\hline & Activity & L1 & $\mathrm{L} 2$ & L3 & \\
\hline \multirow{3}{*}{$\begin{array}{l}\text { Level } 1 . \\
\text { Interpret } \\
\text { the } \\
\text { evidence }\end{array}$} & $\begin{array}{l}\text { Read material to stay current on } \\
\text { emerging research. }\end{array}$ & $\checkmark$ & $\checkmark$ & $\checkmark$ & $\checkmark$ \\
\hline & $\begin{array}{l}\text { Use critical thinking to interpret } \\
\text { implications of research on current } \\
\text { project. }\end{array}$ & $\checkmark$ & $\checkmark$ & $\checkmark$ & $\checkmark$ \\
\hline & $\begin{array}{l}\text { Collect success stories and historical } \\
\text { data on completed projects. }\end{array}$ & $\checkmark$ & $\checkmark$ & $\checkmark$ & $\checkmark$ \\
\hline \multirow{3}{*}{$\begin{array}{l}\text { Level } 2 . \\
\text { Hypothesiz } \\
\text { e and } \\
\text { measure }\end{array}$} & $\begin{array}{l}\text { Perform applied research as a } \\
\text { practitioner on real projects. }\end{array}$ & & $\checkmark$ & $\checkmark$ & 1 \\
\hline & $\begin{array}{l}\text { Hypothesize intended results of } \\
\text { design interventions. }\end{array}$ & & $\checkmark$ & $\checkmark$ & $\checkmark$ \\
\hline & $\begin{array}{l}\text { Measure the results associated } \\
\text { with design interventions. }\end{array}$ & & $\checkmark$ & $\checkmark$ & $\checkmark$ \\
\hline \multirow{3}{*}{$\begin{array}{l}\text { Level } 3 . \\
\text { Share } \\
\text { results } \\
\text { publicly }\end{array}$} & $\begin{array}{l}\text { Report unbiased project results in } \\
\text { the public arena, writing and speaking. }\end{array}$ & & & $\checkmark$ & $\checkmark$ \\
\hline & $\begin{array}{l}\text { Perform independent 3rd party } \\
\text { postoccupancy evaluations. }\end{array}$ & & & $\checkmark$ & $\checkmark$ \\
\hline & $\begin{array}{l}\text { Improve understanding of research } \\
\text { methods through advanced education. }\end{array}$ & & & $\checkmark$ & $\checkmark$ \\
\hline \multirow{3}{*}{$\begin{array}{l}\text { Level } 4 . \\
\text { Meet } \\
\text { academic } \\
\text { standards }\end{array}$} & $\begin{array}{l}\text { Collaborate with credible academic } \\
\text { researchers and social scientists. }\end{array}$ & & & & $\checkmark$ \\
\hline & $\begin{array}{l}\text { Publish research results in peer- } \\
\text { reviewed journals. }\end{array}$ & & & & $\checkmark$ \\
\hline & $\begin{array}{l}\text { Write academic thesis or dissertation } \\
\text { on evidence-based design topic. }\end{array}$ & & & & 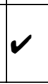 \\
\hline
\end{tabular}

\subsection{Problems in EBD}

주로 프로페션의 실무를 중심으로 번성하고 있는 근거기반 (Evidence-Based) 실무에 대한 다음과 같은 문제점 지적도 발 견된다. 즉, 근거기반의 정체와 유용성을 구체적으로 설명할 수 있는 실질적인 내용은 결핍되어 있음에도 불구하고 하나 의 유행처럼 퍼져가고 있다는 경계의 목소리가 그것이다. 따 라서 근거기반 실무는 새로울 것이 없는 실무관행을 그럴 듯 하게 새로운 것처럼 포장해내는 신조어에 지나지 않는다고 폄하된다(Beam, 2011). 흥미롭게도 의학계에서 대두되는 $\mathrm{EBM}$ 에 관한 비판은 다음과 같은 점에 의해서 건축설계의 지 나친 과학화에 대한 건축계의 경계와 유사한 논거를 갖고 있 다(Lambert, 2006: 2634-2636). 첫째, EBM에서 전체집단에
대한 증거의 표준적 속성에 의해 증거를 개별적 환자의 필요 에 대응하여 적용하기가 어렵다. 둘째, EBM은 통계적 일반화 를 성취한 보편적 증거의 사용을 강조하기 때문에 의사의 개 인화 된 치료방법에 대한 부정적 인식이 있다. 셋째, 임상 경 험을 통해 개인적으로 습득한 지식을 배제하고 있다. 넷째, 형 식화 된 가이드라인의 준수를 강제한다. 다섯째, 환자 개인의 성향에 기인하는 정서적 관점의 차이를 고려하지 못한다. 마 지막으로, EBM 연구에 의한 증거의 통계적 보편성 때문에 환 자별로 존재하는 개인적 편차를 고려해야 하는 임상에 적용 하기 어렵다. 즉, 실무자에 비해 연구자는 이론, 모델, 연구와 객관적 증거를 개념화의 도구로 삼기 때문에(Newman et. al., 2008: 238), 새로운 지식의 창출보다는 기존 지식을 현실적 문제점 해결을 위해 응용하는 임상은, 건축실무와 마찬가지 로, 전문지식을 실무에 적용할 때 발생하는 보편적 지식과 개 별적 지식간의 갈등이 존재하여 $\mathrm{EBM}$ 의 효율성이 의문시 되 고 있다. 따라서 실무적 맥락으로 보면 근거기반모델을 옹호 하는 연구자와 실무자 사이에 가치의 차이가 존재한다.

$\mathrm{EBD}$ 장애요소로는 연구방법론 교육의 부재에 의해 발생한 $\mathrm{EBD}$ 역량을 갖고 있는 건축가의 부족과 $\mathrm{EBD}$ 적용을 위해 소 요되는 투자비용의 문제가 언급된다(Kroll, 2005: 18-20). $\mathrm{EBD}$ 의 개념은 폭 넓게 보급되어 건축계에 널리 알려졌지만 $\mathrm{EBD}$ 증거를 평가하여 실제로 $\mathrm{EBD}$ 에 적용한 설계는 찾아보기 어렵다. 또한 $\mathrm{EBD}$ 의 결과를 적용하기 위해서는 초기 투자자 본의 막대한 증가를 수반한다. 이에 대해 $\mathrm{EBD}$ 는 초기투자 비 용을 증가시키지만 의료사고와 리빌딩에 의한 손실을 감소시 켜 초기 투자의 증가분을 상쇄시킬 수 있다는 연구결과가 있 다(Sadler, et. al., 2008: 22-39).

학부에서부터 높은 수준의 연구방법에 대한 교육적 배경을 가지고 있는 의학분야는 건축교육과는 학제적 지향점의 차이 가 존재한다. 증거의 위계에 대해 다양한 관점의 논쟁을 진행 해 온 $\mathrm{EBM}$ 보다 늦게 형성된 $\mathrm{EBD}$ 는 상대적으로 증거의 유형 에 대해 유연한 입장을 견지하고 있다(Viets, 2009: 76)(표 4).

[Table 4] Comparison of Evidence-Based Practices in Design and Medicine

\begin{tabular}{|c|c|c|}
\hline Comparison Category & Evidence-based medicine (EBM) & Evidence-based design (EBD) \\
\hline First formal recognition & 1992 & 2003 \\
\hline $\begin{array}{l}\text { Types of research } \\
\text { literature }\end{array}$ & $\begin{array}{l}\text { Quantitative methods (including systematic reviews, } \\
\text { meta-analysis, and randomized controlled trials) rank } \\
\text { highest on the evidence pyramid. }\end{array}$ & $\begin{array}{l}\text { Qualitative methods are common; quantitative } \\
\text { methods are also used; few randomized } \\
\text { controlled trials, systematic reviews, or } \\
\text { meta-analyses. }\end{array}$ \\
\hline $\begin{array}{l}\text { Quantity of literature } \\
\text { available }\end{array}$ & $\begin{array}{l}\text { Huge and growing - more than } 100,000 \text { articles } \\
\text { published worldwide in the top } 50 \text { biomedical journals } \\
\text { from 1995-2002 (Soteriades et, al., 2006: 29-34). }\end{array}$ & $\begin{array}{l}\text { Relatively small, but growing body of } \\
\text { rigorous studies relating to healthcare } \\
\text { design (Ulrich et. al., 2008: 61-125). }\end{array}$ \\
\hline Education & $\begin{array}{l}\text { Research skills, methods, and concepts are ingrained } \\
\text { in undergraduate and post-graduate education. }\end{array}$ & $\begin{array}{l}\text { Limited but growing emphasis on research } \\
\text { skills in design education. }\end{array}$ \\
\hline Level of rigor & $\begin{array}{l}\text { Relatively high level of rigor in EBM because the } \\
\text { education system teaches clinicians how to read, } \\
\text { evaluate, and apply literature as well as how to } \\
\text { conduct their own research. }\end{array}$ & $\begin{array}{l}\text { Rigor is often misunderstood by designers. } \\
\text { This may improve as the design community } \\
\text { becomes more informed about EBD } \\
\text { methods. }\end{array}$ \\
\hline
\end{tabular}




\section{EBD Research Trends in Korea}

\subsection{Healthcare Research Trends in Korea}

국내에서 의료건축에 관한 연구결과는 1995년에 창간된 한국의료복지건축학회 논문집을 중심으로 하여 발표되고 있 다. 창간호부터 2013년 8월까지 총 369편의 학술논문이 게재 되어 있다. 의료복지건축학회 논문집에 게재된 논문에서 검색 한 총 1,526 개의 키워드를 대상으로 연구의 초점을 분석하면 공간배치, 공간프로그램, 간호동선 등과 같이 기능과 연계된 총 927 개의 키워드가 발견되어 전체의 $60.74 \%$ 를 차지하고 있다. 다음으로 과학적 연구에 관한 것이 212개(13.89\%) 그리 고 의료복지 정책에 관련된 키워드가 189 개(12.39\%)의 순으 로 발표되어 있다. 기타 게재논문의 키워드에 나타난 연구 초 점은 기술, 운영/관리, 미학 등을 포함하고 있다(표 5).

한편 게재 논문에서 가장 빈번히 발견되는 연구방법3)은 122 편의 논문(33.06\%)에서 발견되는 현장조사로 나타났다. 다음으로 많이 발견되는 연구방법은 각각 108편(29.27\%)과 84편(22.76\%)의 게재논문에서 사용된 사례조사와 문헌조사 이다. 과학적 연구 중 가장 높은 수준의 내적 타당도(Internal Validity)를 성취할 수 있는 (유사)실험연구나 시뮬레이션 연구 는 11 편으로 전체의 $2.98 \%$ 에 지나지 않는 것으로 나타났다 (표 6).

\subsection{Characteristics of EBD Research Trends in Korea}

한국에 $\mathrm{EBD}$ 개념이 리포트의 형식으로 의료복지건축학회 논문집에 2008년 최초로 소개된 이후(Park, 2008: 57-65)4), 키 워드에 $\mathrm{EBD}$ 를 포함한 학술논문은 의료복지건축학회 논문집에 7편, 대한건축학회논문집에 1편 등 총 8편이 발견된다(표 7).

연구방법에 의해 분류하면, 가장 많이 사용된 연구방법은 문헌조사로 4편이 발견되고, 다음으로는 현장조사 2편이 발 견된다. 사례연구와 시뮬레이션 연구는 각각 1 편씩 발견된다. 연구의 효용으로는 해외의 EBD 연구 사례를 예시하여 국내 적용 시 참조점의 역할을 기대하는 기초자료 제시 유형이 3 편, $\mathrm{EBD}$ 방법론을 포함하는 $\mathrm{EBD}$ 연구에 관한 체계적 문헌조 사가 3 편, 과학적 연구 절차에 의해 $\mathrm{EBD}$ 에 적용할 수 있는 근 거를 산출하는 실증적 연구 1편, 그리고 건강한 건축 및 도시 환경 조성에 기여할 수 있는 $\mathrm{EBD}$ 의 거시적 효용을 언급하는 정책연구 1 편이 발견된다.

첫째, 국내 의료건축 연구와 관련하여 가장 많이 발견되는 $\mathrm{EBD}$ 연구 방법은 문헌조사이다. EBD 연구의 소개를 주목적으 로 하는 기초연구의 성격을 갖는 체계적 문헌조사 3 편과 관련 문헌을 조사하여 건강한 환경 구축을 제안하는 정책연구 1편 이 발견된다. 먼저, 미국의 EBD 연구문헌을 분석하여 국내 연 구사례들과 비교할 수 있는 기초자료의 제시를 목적으로 진

[Table 5] Research Focus

\begin{tabular}{l|r|r|r|r|r|r}
\hline \multicolumn{1}{c|}{ Research Focus } & \multicolumn{1}{c|}{1995} & $1996-2000$ & $2001-2005$ & $2006-2010$ & \multicolumn{1}{c}{$2011-2013$} & \multicolumn{1}{c}{ Sum } \\
\hline Function & $33(67.35)$ & $246(60.00)$ & $199(62.97)$ & $289(58.98)$ & $160(61.30)$ & $927(60.74)$ \\
\hline Scientific research & $4(8.16)$ & $46(11.22)$ & $37(11.71)$ & $70(14.29)$ & $55(21.07)$ & $212(13.89)$ \\
\hline Policy & $2(4.08)$ & $61(14.88)$ & $29(9.18)$ & $65(13.27)$ & $32(12.26)$ & $189(12.39)$ \\
\hline Others $*$ & $10(20.41)$ & $57(13.90)$ & $51(16.14)$ & $66(13.47)$ & $14(5.37)$ & $198(12.98)$ \\
\hline Grand Sum & $49(100 \%)$ & $410(100 \%)$ & $316(100 \%)$ & $490(100 \%)$ & $261(100 \%)$ & $1,526(100 \%)$ \\
\hline
\end{tabular}

* Others include several items such as technology, maintenance/operation, and aesthetics.

[Table 6] Research Method

\begin{tabular}{l|r|r|r|r|r|r}
\hline \multicolumn{1}{c|}{ Research Method } & \multicolumn{1}{c|}{1995} & $1996-2000$ & \multicolumn{1}{c|}{$2001-2005$} & \multicolumn{1}{c}{$2006-2010$} & \multicolumn{1}{c}{$2011-2013$} & \multicolumn{1}{c}{ Sum } \\
\hline Field study & $9(81.82)$ & $34(36.17)$ & $34(40.00)$ & $25(21.19)$ & $20(32.79)$ & $122(33.06)$ \\
\hline Case study & $1(9.09)$ & $30(31.91)$ & $24(28.24)$ & $40(33.90)$ & $13(21.31)$ & $108(29.27)$ \\
\hline Literature review & $1(9.09)$ & $21(22.34)$ & $15(17.65)$ & $31(26.27)$ & $16(26.23)$ & $84(22.76)$ \\
\hline $\begin{array}{l}\text { Pseudo)experimental } \\
\text { research/simulation }\end{array}$ & $0(0.00)$ & $2(2.13)$ & $3(3.53)$ & $3(2.54)$ & $3(4.92)$ & $11(2.98)$ \\
\hline Others $*$ & $0(0.00)$ & $7(7.45)$ & $9(10.59)$ & $19(16.10)$ & $9(14.75)$ & $44(11.92)$ \\
\hline Grand Sum & $11(100 \%)$ & $94(100 \%)$ & $85(100 \%)$ & $118(100 \%)$ & $61(100 \%)$ & $369(100 \%)$ \\
\hline
\end{tabular}

* Others include several items such as survey and action research.

3) 복수연구방법을 채택한 연구논문의 분류는 논의 전개에 가장 큰 영향을 미치는 증거의 수집과 관련된 주요 연구방법을 지칭한다.
4) 이 보고서는 $\mathrm{EBD}$ 와 관련된 중요한 이론적 근거 중의 하나인 지원 이론(supportive design theory)과 $\mathrm{EBD}$ 의 사례를 소개하고 있다. 
[Table 7] Characteristics of EBD-Related Research Articles in Korea

\begin{tabular}{|c|c|c|c|}
\hline Titles & Methods & Utility & Keywords \\
\hline $\begin{array}{l}\text { A study on healing environments and evidence-based } \\
\text { design, Journal of Korea Institute of Healthcare } \\
\text { Architecture, Vol.17, No.1, 2011. }\end{array}$ & $\begin{array}{l}\text { literature } \\
\text { review }\end{array}$ & $\begin{array}{l}\text { systematic } \\
\text { literature } \\
\text { review }\end{array}$ & $\begin{array}{l}\text { evidence-based design, healthcare facility } \\
\text { planning, healing environment }\end{array}$ \\
\hline $\begin{array}{l}\text { A study on healing environmental factors that promote } \\
\text { safety, quality of life, and health of elderly patient, } \\
\text { Journal of Korea Institute of Healthcare Architecture, } \\
\text { Vol.19, No.3, 2013. }\end{array}$ & $\begin{array}{l}\text { literature } \\
\text { review }\end{array}$ & $\begin{array}{l}\text { systematic } \\
\text { literature } \\
\text { review }\end{array}$ & $\begin{array}{l}\text { geriatric hospitals, evidence-based design, } \\
\text { healing environments, health }\end{array}$ \\
\hline $\begin{array}{l}\text { A methodological considering on the evidence-based } \\
\text { design related to health effects, Journal of Korea } \\
\text { Institute of Healthcare Architecture, Vol.17, No.2, } 2011 .\end{array}$ & $\begin{array}{l}\text { literature } \\
\text { review }\end{array}$ & $\begin{array}{l}\text { systematic } \\
\text { literature } \\
\text { review }\end{array}$ & $\begin{array}{l}\text { evidence-based design, health effect, } \\
\text { methodology, measurement }\end{array}$ \\
\hline $\begin{array}{l}\text { A study on the research model for healthy urban and } \\
\text { architectural environment, Journal of Korea Institute of } \\
\text { Healthcare Architecture, Vol.19, No.1, } 2013 \text {. }\end{array}$ & $\begin{array}{l}\text { literature } \\
\text { review }\end{array}$ & policy & $\begin{array}{l}\text { health, architectural planning for health, } \\
\text { healthy city environment, ecological } \\
\text { environment, evidence-based design, health } \\
\text { care environment }\end{array}$ \\
\hline $\begin{array}{l}\text { The evaluation of planning elements in the senior } \\
\text { housing environment based on characteristics of elderly, } \\
\text { Journal of the Architectural Institute of Korea, Vol.27. } \\
\text { No.9, 2011. }\end{array}$ & field & $\begin{array}{l}\text { preliminary } \\
\text { EBD } \\
\text { research }\end{array}$ & $\begin{array}{l}\text { senior housing environment, characteristics } \\
\text { of elderly, evidence-based design }\end{array}$ \\
\hline $\begin{array}{l}\text { A study on the healing rest space in pediatric ward, } \\
\text { Journal of Korea Institute of Healthcare Architecture, } \\
\text { Vol.19, No.1, 2013. }\end{array}$ & field study & $\begin{array}{l}\text { preliminary } \\
\text { EBD } \\
\text { research }\end{array}$ & $\begin{array}{l}\text { children's hospital, healing environments, } \\
\text { rest space, evidence-based design }\end{array}$ \\
\hline $\begin{array}{l}\text { A case study on extension design of Seoul National } \\
\text { University Bundang hospital, Journal of Korea Institute } \\
\text { of Healthcare Architecture, Vol.16, No.3, 2010. }\end{array}$ & case study & $\begin{array}{l}\text { preliminary } \\
\text { EBD } \\
\text { research }\end{array}$ & $\begin{array}{l}\text { extension, context, hospital street, space } \\
\text { design marketing, hospital image, evidence- } \\
\text { based design }\end{array}$ \\
\hline $\begin{array}{l}\text { Korean pediatric patients' preferences for patient room } \\
\text { design, Journal of Korea Institute of Healthcare } \\
\text { Architecture, Vol.17, No.2, 2011. }\end{array}$ & simulation & $\begin{array}{l}\text { scientific } \\
\text { research }\end{array}$ & $\begin{array}{l}\text { children's color preferences, pediatric patient } \\
\text { room, healing environment, simulation, } \\
\text { evidence-based design }\end{array}$ \\
\hline
\end{tabular}

행된 연구문헌에서는 관련 문헌에 나타난 $\mathrm{EBD}$ 의 특성을 소 개한 후: (1) 환자를 위한 환경; (2) 의료진을 위한 환경; 그리 고 (3) 스트레스를 주는 환경에 대한 EBD 연구를 분석하여 치유환경 설계에 적용할 수 있는 근거자료를 소개하고 있다. 과학적 방법론을 사용한 연구결과를 $\mathrm{EBD}$ 근거체계의 상위 에 두고 있다(Park, 2011: 41-50).

또 다른 체계적 문헌조사 연구 유형으로 노인전문병원과 노인요양시설을 대상으로 하여 노인의 안전과 삶의 질에 영 향을 미치는 치유환경요소를 분석한 연구가 있다. 구체적으로 는 치유환경요소와 치유효과의 관계에 관한 실증적 연구문헌 을 분석하여 치유환경이 제공하는 치유효과를 다음과 같이 요약하고 있다: (1) 약물사용 감소; (2) 낙상 감소; (3) 배회 감 소; (4) 삶의 질 향상; (5) 웰빙 향상; (6) 수면의 질 향상; (7) 불안 행동 감소; (8) 인지적 및 신체적 수행능력 증진; (9) 방향 감과 길찾기 능력 개선; 그리고 (10) 신체 활동의 증가(Choia), 2013: 7-19).

$\mathrm{EBD}$ 관련 연구의 방법론에 초점을 두고 진행한 체계적 문 헌조사에서는 과학적 근거에 대한 측정방법의 유형과 성격을 분류하여 $\mathrm{EBD}$ 연구의 방법론적 지향점을 제시하고 있다. 연 구자는 $\mathrm{EBD}$ 는 인과적 설명을 토대로 대안적 설명을 허용하지
않는 실증적 설계기준을 제시하는 것으로 파악하고 있다. 문 헌조사를 통해 EBD 방법을 고찰한 후 외과 수술 후 회복 환자 를 대상으로 한 설문조사를 통해 자연환경이 주는 치유효과 에 대한 인지도를 조사하고 환자의 치료 및 회복기록을 참조 하여 회복 효과에 대한 신뢰도를 평가하려는 시도를 하고 있 다. 이러한 과정을 통해 $\mathrm{EBD}$ 정착을 위해서는 신뢰도 높은 근 거를 산출할 수 있는 임상과학적 연구방법론의 적용이 필요 하다는 제안을 하고 있다(Min, 2011: 35-44).

마지막으로 발견되는 $\mathrm{EBD}$ 와 관련된 문헌조사의 유형으로 는 건강한 건축 및 도시환경을 조성하기 위한 연구 모형을 제 시하고 있는 거시적 관점의 이론 연구가 있다. 특히, 건강한 건축 및 도시 구축에 미치는 여러 요인 중에서 $\mathrm{EBD}$ 의 적용이 기여할 수 있는 긍정적 영향을 정책적 관점에서 논하고 있다 (Choib), 2013: 35-46).

둘째, 국내 의료건축 연구에서 문헌조사 다음으로 많이 발 견되는 $\mathrm{EBD}$ 와 관련된 연구방법은 $\mathrm{EBD}$ 의 관점으로 의료복지 시설을 평가하는 현장조사이다. 먼저, 노인을 위한 스마트 홈 구축을 위한 가이드라인 제시를 목적으로 하는 기초연구에서 는 총 61 편의 국내외 문헌을 조사하여 고령친화 주거공간의 계획요소를 추출하고 있다. 이를 토대로 건축관련 전문가를 
대상으로 하는 고령친화 주거공간의 계획요소 중요도 인식 설문조사를 병행하고 있다. 방법론적으로 보면 전문가 설문조 사를 통해 건축개념과 계획요소의 관련성을 조사한 후 적용 성 정도를 평가하기 위해 서울과 뉴욕에 위치한 고령자 주택 및 시설을 각각 2곳 씩 현장 방문하여 관련 자료를 수집하였 다. 7 개의 고령친화적 건축개념(감각자극, 안전성, 사회성, 편 리성, 개인화, 접근성, 적응성)의 적용실태를 평가하여 조사대 상지에서는 적응성 확보가 가장 미흡함을 밝히고 있다. 방법 론상의 특징은 설문조사를 통해 분석한 건축전문가의 의견을 고령자 주택의 주거환경 계획요소의 적절성을 판단하는 근거 로 사용하는 점에서 발견된다(Kim et. al., 2011: 151-160).

또한 소아과 병동 휴게공간의 치유환경을 $\mathrm{EBD}$ 관점으로 평가한 현장조사연구가 있다. 휴게공간의 치유적 효과를 평가 하는 근거자료는 서울에 위치한 종합병원의 소아과 병동 3곳 을 현장조사 하여 수집하였다. 또한 휴게공간의 적절성에 대 한 의견을 묻는 설문조사를 어린이 환자의 보호자를 대상으 로 하여 실시하였다(Jung et. al., 2013: 23-34). 이러한 절차 를 통해 어린이 병동의 치유적 휴게공간 계획 방안을 제언하 고 있다.

셋째, 국내 EBD 연구에서 발견되는 또 다른 연구방법으로 대학병원의 증축 프로젝트를 대상으로 한 사례연구가 있다. 병원설계와 관련된 문헌, 기본 계획안과 입찰 안내서 등을 분 석한다. 이러한 자료에 나타난 사용자의 요구사항, 스페이스 프로그램, 설계 전단계에서 행해진 현황조사의 분석을 통해 병원 증축 설계에 참조점을 제공하는 것을 연구의 목적으로 하고 있다. EBD에서 디자인 결정의 근거로 사용할 수 있는 증 거를 도출하기 위한 구체적 방법론을 살펴보면, 조사 병원의 증축 용도별 면적 비율, 스페이스 프로그램, 설계기본개념(조 망, 옥상정원 등), 배치계획, 동선 계획, 병동부 계획, 중앙진료 부 계획, 외래진료부 계획, 공용공간 계획, 친환경계획 등에 관한 2차 자료를 사용하여 계획요소의 특성을 기술하고 있다. 따라서 $\mathrm{EBD}$ 는 여러 분석 단위 중의 하나로 소개되어 있기 때 문에 $\mathrm{EBD}$ 에 집중한 연구라기보다는 종합병원 증축에 $\mathrm{EBD}$ 의 적용 필요성을 제시하는 사례연구로 보는 것이 타당하다 (Park; Moon, 2010: 27-35).

마지막으로, 한국 소아과 병실 설계에 적용할 수 있는 $\mathrm{EBD}$ 연구의 일환으로 어린이 환자의 색채선호도 평가에 대한 실 증적 연구가 있다. 구체적으로 보면, 소아병원의 병실 색채를 분석의 단위로 설정하고 모형을 활용한 시뮬레이션 연구방법 이 사용되었다(Park; Park, 2011: 45-52). 50명(남아 30명과 여아 20명)을 대상으로 1:12 스케일의 병실 모형을 이용한 시 뮬레이션으로 색채선호도를 조사하였다. 독립변수로는 빨간 색, 노란색, 초록색, 파란색, 보라색, 그리고 하얀색을 사용하 였고 종속변수로는 어린이 환자의 색채 선호도를 설정하여 측정하였다. 측정 결과를 활용하여 통계적 기법으로 색채 선
호도를 분석하여 소아병동 색채계획에 관한 증거를 산출하고 있다.

\section{Characteristics of EBD Research in Korea}

국내에서 발견되는 대부분의 $\mathrm{EBD}$ 연구는 과학적 연구 디 자인에 의해 이루어지지 않고 있어, 최선의 실증적 증거에 기 반을 둔 설계 결정이 제공하는 긍정적 치유효과에 대한 경험 적 논의는 제한적이다. $\mathrm{EBD}$ 방법론에 대한 교육과 정보의 부 족이 $\mathrm{EBD}$ 의 실무적 확산에 미치는 부정적 영향을 인지한 미 국 건축계의 경우, 전문교육프로그램을 운영하고 있는 대학의 연구자를 중심으로 하여 실증적 연구뿐만이 아니라 과학적으 로 진행한 경험적 연구를 체계적으로 분석한 다양한 연구가 발표되고 있다(Ulrich, R.S. et. al., 2008: 61-125). 또한 실무적 관점에서 $\mathrm{EBD}$ 역량을 검증하기 위해 $\mathrm{CHD}$ 가 주관하는 $\mathrm{EDAC}$ 자격시험을 통해 $\mathrm{EBD}$ 와 관련된 지식 저변을 확대하는 노력을 하고 있다.

한국의료복지건축학회 논문집을 통해 국내에 소개되어 점 차 주목을 받고 있는 $\mathrm{EBD}$ 의 방법론적 스펙트럼은 아직 불균 형적 현황을 노출하고 있다. 2008년 이후, 국내 건축논문집에 소개되기 시작한 $\mathrm{EBD}$ 연구는 체계적 기준에 의해 선정한 연 구문헌들을 분석하여 $\mathrm{EBD}$ 의 실증적 효과를 요약하는 연구결 과와 $\mathrm{EBD}$ 가 적용된 외국의 사례를 분석하여 국내 의료건축 설계 평가의 틀이나 가이드라인을 제시하여 치유환경 구축을 위한 도구적 효용을 갖는 현장조사 및 사례연구가 주로 발표 되고 있다. 반면에 EBD 연구에서 가장 높은 위계를 인정받는 무작위 샘플링에 의한 복수의 RCT 연구디자인에 의해 진행한 연구는 국내에서 아직 발견되지 않고 있다. 표 2에 예시된 연 구디자인에 따른 산출 증거의 위계로 국내 $\mathrm{EBD}$ 관련 연구를 평가한다면 총 6 단계 중 3,4 단계의 위계를 갖고 있는 것으로 보인다.

증거의 수준을 평가하기 위해 한국의료복지건축학회 논문 집에 게재된 의료복지건축에 관련된 연구논문과 $\mathrm{EBD}$ 에 관련 된 연구논문을 연구방법의 유형으로 비교해 보면 전체논문에 서는 현장조사(33.06\%)가 가장 많이 사용되었으나 과학적 연 구방법(2.98\%)의 사용빈도는 가장 낮았다. 반면 $\mathrm{EBD}$ 와 관련 해서는 문헌조사(50\%)가 가장 많이 사용되었고 사례연구 (12.5\%)와 과학적 연구(12.5\%)의 사용빈도가 가장 낮았다. 사 례연구의 경우, 전체 연구에서는 두 번째로 많이 사용되고 있 으나(29.27\%), EBD 연구에서는 사용 빈도가 상대적으로 낮게 나타난다. 한국의료복지건축학회 논문집에 게재된 논문 중 과 학적 연구방법의 사용은 제한적이나, $\mathrm{EBD}$ 연구에서는 $12.5 \%$ 로 상대적 사용빈도가 높다(그림 1).

$\mathrm{EBD}$ 연구동향을 분석하는 본 연구의 결과는 현재 국내건 축학계에 발표된 $\mathrm{EBD}$ 와 관련된 연구논문의 수가 많지 않아 
비교분석의 통계적 타당성은 주장할 수 없다. 그러나 $\mathrm{EBD}$ 의 전개 방향과 연구 단계에 대한 개략적인 시사점을 제공하고 있다는 점에서 그 의의가 있다. 즉, 일반적으로 건축학 분야의 연구에서는 주로 질적 연구인 현장조사와 사례연구가 많이 진행되고 있으나, $\mathrm{EBD}$ 와 관련해서는 그 빈도가 상대적으로 낮게 나타나고 있다. 반면에 국내 $\mathrm{EBD}$ 연구에서는 특징적으 로 문헌조사에 의한 연구결과물들이 많이 발표되어 있다. 문 헌조사가 국내 $\mathrm{EBD}$ 연구를 주도하고 있는 현상은 의학에서 시작된 $\mathrm{EBM}$ 의 발전에 영향 받은 의료건축설계 분야가 새로 운 실무 방식으로 고안한 $\mathrm{EBD}$ 의 개념과 연구 사례를 국내에 소개하여 저변을 확장시키는 도입단계에 있기 때문으로 이해 된다.

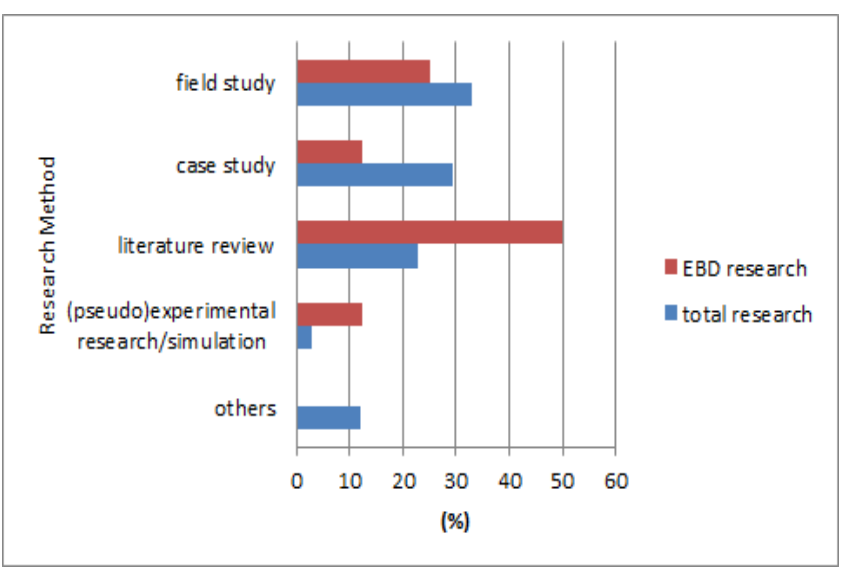

[Figure 1] Comparison of Research Methods

\section{Conclusion}

최근 의료건축설계를 중심으로 번성하고 있는 EBD 연구의 개념과 증거의 질을 평가하는 연구방법의 속성을 중심으로 하여, 국내 EBD 연구경향을 분석한 본 연구는 다음과 같이 결 론짓는다.

첫째, 현재 대부분의 국내 $\mathrm{EBD}$ 연구는 미국 $\mathrm{EBD}$ 연구논문 을 검토 및 소개하는 문헌조사가 많이 발견된다. 의료복지건 축과 관련된 전체 연구논문의 경향과 상이하게 $\mathrm{EBD}$ 연구에서 문헌조사의 사용이 가장 높게 나타나는 현상은 국내 EBD 연 구는 아직 관심 단계에 있다는 반증으로 보인다. 증거의 위계 를 높일 수 있도록 과학적 연구를 포함하는 연구디자인에 의 해 다양한 연구 결과물이 발표되기 시작할 때 국내 EBD 연구 는 성숙기에 접어들었다고 평가할 수 있게 된다.

둘째, 전통적으로 증거의 최상위 위계를 차지하는 과학적 연구디자인에 의해 진행한 연구 빈도가 상대적으로 낮아 향 후 $\mathrm{EBD}$ 연구의 발전 방향점을 제시한다. 즉, 증거의 실무적 효용을 높이기 이해서는 과학적 연구디자인을 포함하는 연구 수행의 필요성이 대두된다.
그러나, 본 연구에서 분석한 국내 $\mathrm{EBD}$ 관련 연구는 양적인 면에서나 방법론적 면에서 제한적이어서 심도 있는 분석에는 한계가 있다. 따라서 국내 EBD 연구 현황, 특성 및 단계를 이 해할 수 있게 하고, 또한 해외 $\mathrm{EBD}$ 연구의 강조점과 지향점에 대한 이해를 통해 국내 EBD 연구의 현황 파악과 향후 전개 방 향 설정을 돕는 기초자료로서의 역할을 기대한다. 초기의 $\mathrm{EBM}$ 이 최고의 증거로서 과학적 연구를 강조하였으나, 실증주 의(Positivism)에 기반을 둔 연구가 추구하는 일반화된 결론은 개인적 환자의 임상적 특이성이나 심리적 니즈에 대응할 수 없다는 한계를 인식하게 되었다. 결국 증거의 스펙트럼이 확 장되어 문맥에 따라 질적 연구에 의한 해석적 접근방식의 중 요성을 강조하는 경향이 대두되고 있다. 따라서 과학적 프로 페션인 의학의 $\mathrm{EBM}$ 과 미학적 프로페션인 건축의 $\mathrm{EBD}$ 간에 존재하는 학제간 증거의 유형에 대한 논의, 즉 과학적 방법론 과 질적 연구방법론의 증거로서의 가치와 근거기반 실무의 절차에 대한 구체적 논의는 다음 연구의 주제로 남겨 놓는다.

\section{References}

Beam, A. Knowledge-based gibberish, The Boston Globe, 2011, http://articles.com/2011-07-08/lifestyle/29752513_1_evidence-ver bal-tcis-reality (accessed 15 July 2013).

Briner; Denyer; Rousseau. 2009, p.19. In Carr, V. et. al., Integrating evidence-based design and experience-based approaches in healthcare service design, Health Environments Research \& Design Journal, Vol.4 No.4, 2011.

Choi, Kwangseokb). A study on the research model for healthy urban and architectural environment, Journal of Korea Institute of Healthcare Architecture, Vol.19, No.1, 2013.

Choi, Young-Seon.a) A study on healing environmental factors that promote safety, quality of life, and health of elderly patient, Journal of Korea Institute of Healthcare Architecture, Vol.19, No.3, 2013.

Douglas, K.; Hathaway, R.; Burks, S. 'The environment matters' and 'designing toward the whole,' Nursing Economics, Vol.29, No.1, 2011.

EBM Working Group. Evidence-based medicine, Journal of the American Medical Association, Vol.268, 1992.

Hamilton, D.K. What constitutes best practice in healthcare design, Health Environments Research \& Design Journal, Vol.4, No.2, 2011.

Hamilton, D.K. Evidence-based design supports evidence-based medicine in the ICU, ICU Management Journal, Vol.6, No.3, 2006.

Jung, So Young; Kim, Ye-Seul; Choi, Kwangseok. A study on the healing rest space in pediatric ward, Journal of Korea Institute of Healthcare Architecture, Vol.19, No.1, 2013.

Kim, Dae-Jin; Shin, Hye-Kyeong; Ryu, Ho-Sik. The evaluation of planning elements in the senior housing environment based on characteristics of elderly, Journal of the Architectural Institute of Korea, Vol.27. No.9, 2011.

Kim, Duk-Su; Shepley, M. Healthcare architects' professional 
autonomy: Interview case studies, Health Environments Research \& Design Journal, Vol.1, No.2, 2008.

Kroll, K. Better health from better design? Building Operating Management, Vol.52, No.1, 2005.

Lambert, H. Accounting for EBM: Notions of evidence in medicine, Social Science \& Medicine, Vol.62, 2006.

Lavizzo-Mourey, R. President's address, Annual Report, Robert Wood Johnson Foundation, Princeton, NJ: Robert Wood Johnson Foundation, 2004.

Lewis, R. Architects to benefit from new tools, Health Facilities Management, October, 2008.

Min, Sang Choong. A methodological considering on the evidence-based design related to health effects, Journal of Korea Institute of Healthcare Architecture, Vol.17, No.2, 2011.

Moon, Changho. A study on the specialization of university education about healthcare facility design in USA, Korean Institute of Healthcare Architecture Journal, Vol.17, No.1, 2011.

Munhall, A. Nursing, research and the evidence. Evidence Based Nursing, Vol. 1, 1998.

Newell-Stokes, G. Evidence-based practice and the role of nursing leadership, Journal of Nursing Administration, Vol. 28, 7/8, 1998.

Newman, M.; Papadopoulos, I.; Sigsworth, J. Barriers to evidence-based practice, Intensive and Critical Care Nursing, Vol.4, No.23, 2008.

Noakes, P. Insights: for the future of healthcare design, Healthcare Design, 2009.11.

Park, Jin Gyu; Park, Changbae. Korean pediatric patients' preferences for patient room design, Journal of Korea Institute of Healthcare Architecture, Vol.17, No.2, 2011.

Park, Jin Gyu. Evidence-based design in healthcare environments, Journal of Korea Institute of Healthcare Architecture, Vol.14, No.3, 2008.

Park, Jin Gyu. A study on healing environments and evidence-based design-Focused on healthcare environments in the United States, Journal of Korea Institute of Healthcare Architecture, Vol.17, No.1, 2011.

Park, Sungsine; Moon, Changho. A case study on extension design of Seoul National University Bundang hospital," Journal of Korea Institute of Healthcare Architecture, Vol.16, No.3, 2010.

Pati, D. A framework for evaluating evidence in evidence-based design, Health Environments Research \& Design Journal, Vol.4, No.3, 2011.

Sadler, B.L.; DuBose, J.; Zimring, C. The business case for building better hospitals through evidence-based design, Health Environments Research \& Design Journal, Vol.1, No.3, 2008.

Saitz, R. Evidence-based design: part of evidence-based medicine? Evidence-Based Medicine, Vol.18, No.1, 2013.

Stetler C.; Brunell M.; Giuliano K.; Morsi D.; Prince L.; Newell-Stokes G. Evidence-based practice and the role of nursing leadership, Journal of Nursing Administration, Vol. 28, 7/8, 1998.

Stichler, J.F. Research or evidence-based design: Which process should we be using? Health Environments Research \& Design Journal, Vol.4 No.1, 2010.

Stichler, J.F. Weighing the evidence. Health Environments Research \&
Design Journal, Vol.3, No.4, 2010.

Stichler, K.F.; Hamilton, D.K. Evidence-based design: What is it?, Health Environments Research \& Design Journal, Vol.1, No.2, 2008.

Soteriades, E. et. al., Research contribution of different world regions in the top 50 biomedical journals(1995-2002). The FASED Journal, Vol.20, No.1, 2006.

Ulrich, R.S. et. al. A review of the research literature on evidence-based healthcare design, Health Environments Research \& Design Journal, Vol.1, No.2, 2008.

Viets, E. Lessons from evidence-based medicine: what healthcare designers can learn from the medical field, Health Environments Research \& Design Journal, Vol.2, No.2, 2009.

접수 : 2014년 03월 01일 1차 심사 완료 : 2014년 04월 19일 게재확정일자 : 2014년 04월 19일 3인 익명 심사 필 